\title{
Self-limiting earthquake dynamics and spatio-temporal clustering of seismicity enabled by off-fault plasticity
}

\author{
Md Shumon Mia ${ }^{1,2}$, Mohamed Abdelmeguid ${ }^{1}$, Ahmed E. Elbanna ${ }^{1,3}$ \\ ${ }^{1}$ Department of Civil and Environmental Engineering, University of Illinois at Urbana-Champaign, Urbana, IL, USA. \\ ${ }^{2}$ Department of Mechanical Science and Engineering, University of Illinois at Urbana-Champaign, Urbana, IL, USA. \\ ${ }^{3}$ Beckman Institute of Advanced Science and Technology, University of Illinois at Urbana-Champaign, Urbana, IL, USA
}

This is a non-peer reviewed preprint submitted to EarthArXiv.

\begin{abstract}
Earthquakes are among nature's deadliest and costliest hazards. Understanding mechanisms for earthquake nucleation, propagation, and arrest is key for developing reliable operational forecasts and next generation seismic hazard models. While significant progress has been made in understanding source processes in linear elastic domains, the response of the rocks near the fault is complex and likely to be inelastic due to the extreme stresses and deformations associated with fault slip. The effect of this more realistic fault zone response on seismic and aseismic fault slip is poorly understood. Here, we simulate sequence of earthquake and aseismic slip of a fault embedded in an elastic-viscoplastic bulk subject to slow tectonic loading. We show that off-fault plasticity significantly influences the source characteristics. Specifically, off-fault plasticity may lead to partial ruptures and emergence of spatial segmentation as well as hierarchical temporal seismic clustering. Furthermore, co-evolution of fault slip and off-fault bulk plasticity may lead to heterogeneous rupture propagation and results in pockets of slip deficit. While the energy dissipated through plastic deformation remains a small fraction of the total energy budget, its impact on the source characteristics is disproportionally large through the redistribution of stresses and viscous relaxation. Our results suggest a new mechanism of dynamic heterogeneity in earthquake physics that can be active for both small and large earthquakes and may have important implications on earthquake size distribution and energy budget. Furthermore, this plasticity-induced self-limiting crack dynamics may be relevant for other dynamic fracture applications and design of dynamically tough materials.
\end{abstract}

\section{Introduction}

Earthquakes are a prime example of a complex natural processes with far-from-equilibrium strongly nonlinear dynamics, having substantial societal and economic relevance for large populations worldwide. The lack of quantitative data on timescales capturing multiple large earthquake cycles is a long-standing challenge. Physics-based simulations provide the only path for overcoming the lack of data and elucidating multi-scale dynamics and spatio-temporal patterns that extend the knowledge beyond sporadic case studies and regional statistical laws.

The intrinsic multiscale nature of the physical processes that control stable and unstable fault slip is widely acknowledged. Spatial scales may range from a sub-meter process zone near the crack 
tip to tens or hundreds of kilometers of fault length. Temporal scales ranges from years of slow slip associated with tectonic loading to few seconds in co-seismic rapid failure. This multiscale nature of the problem has been a major challenge in the field. While significant progress has been made in modeling single instances of dynamic earthquake ruptures, modeling a single rupture may not be sufficient to get insights into the full history of fault slip. This is because results of single event simulations involve artificial nucleation and depend critically on the prescribed initial conditions. Modeling Sequence of Earthquake and Aseismic Slip (SEAS) is necessary to illuminate pattern of earthquake cycles over long time as well as evolution of fault slip both during seismic rupture and inter-seismic interval.

The majority of previous SEAS studies have been conducted either with fully elastic bulk or limited to the quasi-dynamic approximation where inertia effects, crucial for the dynamic phase, are not fully considered but only approximated through radiation damping. Earthquake sequences for a planar fault with elastic bulk usually exhibit a periodic pattern. A mixture of large and small events may arise on planar faults in purely elastic bulk given sufficient quenched heterogeneity, e.g., variability in frictional properties (Lapusta et al., 2000; Lapusta \& Rice, 2003; Kaneko et al., 2010) or incorporation of a compliant fault zone relative to the host rock (Abdelmeguid et al., 2019; Thakur et al. 2020) or if the fault is too long compared to the characteristic frictional-elastic length scale (Cattania, 2019; Barbot, 2019). Very few studies have considered earthquake sequence simulations on non-planar faults. Most notably, Cattania and Segall (2021) showed that within the quasidynamic approximation limit, non-planar faults having long wavelength fractal roughness may generate foreschocks and microseismicity. Yuval et al. (2017) also studied the effect of fault roughness on earthquake nucleation using a fully dynamic simulation but assuming the bulk remain linear elastic.

Only a handful of studies investigated the effect of bulk material response, beyond elasticity, on sequence of earthquake and aseismic slip. These include cycle simulation with bulk viscoelasticity approximating inertia with radiation damping (Allison \& Dunham, 2018, 2021) as well as full account of inertia (Duru et al., 2019). Temperature dependent viscous flow has recently been considered and is shown to reduce the creeping of the velocity strengthening portion of the fault (Allison \& Dunham, 2021). While it has long been recognized, using numerical simulations of single dynamic rupture events, that off-fault plasticity acts as an energy sink and influences the rupture characteristics by reducing peak slip rate and limiting rupture speed (Templeton \& Rice, 2008; Viesca et al., 2008; Dunham et al. 2011a, 2011b; Gabriel et al. 2013), only one study so far, to the best of our knowledge, has investigated the effect of off-fault plasticity in cycle simulation (Erickson et al., 2017). This study, however, was limited to the quasi-dynamic approximation and showed that the pattern of seismic cycle is like the reference elastic case except for slip deficit near free surface. It is not clear whether this insignificant difference between the plastic and elastic cases is intrinsic or is a consequence of the choice of the parameters and approximations involved. This is critical to investigate since natural fault zones exhibit significant inelasticity and their evolution over short and long-time scales ought to be playing a role in the source physics.

Here, we investigate, for the first time to the best of our knowledge, the effect of off-fault plasticity throughout the earthquake cycle for a 2D anti-plane rate-and-state fault using a novel hybrid finite element-spectral boundary integral scheme that alternates between dynamics and 
quasi-dynamics solver accounting for the full inertia effect. This computationally efficient numerical scheme enables high resolution modeling of fault zone inelastic response and allows for exploration of parameters over wider range than what was possible before. Using this technique, we explore the phase space of earthquake events as the off-fault bulk properties, namely the yield strength and viscous relaxation time scales, are varied. We identify a possible transition from simple periodic events to spatio-temporal clustering of seismicity marked by a boundary that is defined by a competition between the relaxation time scale and a frictional time scale on one hand, and the yield stress and elastic nucleation stress on the other hand. We outline the setup of the model and summarize the main results in the next sections.

\section{Model setup}

We consider a 2D anti-plane fault containing seismogenic velocity-weakening (VW) and creeping velocity strengthening (VS) patches (Fig-1). Out-of-plane loading is applied in the form of constant plate rate, $V_{p}=35 \mathrm{~mm} /$ year $\approx 10^{-9} \mathrm{~m} / \mathrm{s}$. List of parameters used in the simulations are summarized in Supplementary Materials (Table-S1). The fault friction is governed by a regularized rate-and-state friction (Dieterich, 1979; Ruina, 1983; Ben-Zion \& Rice, 1997; Lapusta et al., 2000) where the friction coefficient, $f$, is a function of slip rate and state variable:

$$
f(V, \theta)=\operatorname{asinh}^{-1}\left[\frac{V}{2 V_{0}} \exp \left(\frac{f_{0}+b \ln \left(V_{0} \theta / L\right)}{a}\right)\right] .
$$

Here, $a$ and $b$ are nondimensional frictional parameters related to direct effect and state evolution respectively. $a-b<0$ indicates velocity weakening friction whereas $a-b>0$ indicates velocity strengthening friction. $L$ is critical slip weakening distance and $\theta$ is state variable which evolves following a prescribed aging law (Ruina, 1983; Ampuero \& Rubin, 2008):

$$
\frac{d \theta}{d t}=1-\frac{V \theta}{L} .
$$

We consider constant effective normal stress, $\sigma_{n}$. The fault strength is then expressed as $\sigma_{n} f(V, \theta)$. Off-fault bulk constitutive response is elastic-viscoplastic. We consider J2 plasticity model with viscous regularization. For 2D anti-plane problem, it reduces to a yield function:

$$
F(\sigma)=\sqrt{\left(\sigma_{x z}^{2}+\sigma_{y z}^{2}\right)}-\sigma_{y}
$$

Where, $\sigma_{y}$ is the bulk yield strength. With Perzyna type viscoplastic relation (Simo \& Hughes, 2006), viscoplastic strain rate is expressed as,

$$
\begin{gathered}
\dot{\epsilon}_{x z}{ }^{v p}=\gamma \frac{\partial F}{\partial \sigma_{x z}}, \text { and } \dot{\epsilon}_{y z} v p=\gamma \frac{\partial F}{\partial \sigma_{y z}} \\
\text { with } \gamma=\frac{\langle F(\sigma)\rangle}{\eta}
\end{gathered}
$$

where, $\eta$ denotes viscosity. Bulk constitutive relation for the 2D anti-plane problem, assuming an additive decomposition of the strain, then follows:

$$
\sigma_{x z}=2 \mu\left(\epsilon_{x z}-\epsilon_{x z}^{v p}\right), \text { and } \quad \sigma_{y z}=2 \mu\left(\epsilon_{y z}-\epsilon_{y z}^{v p}\right) \quad \text { (Eqn-6a, 6b) }
$$


where, $\mu$ is the shear modulus and $\epsilon_{i j}$ indicates total strain.
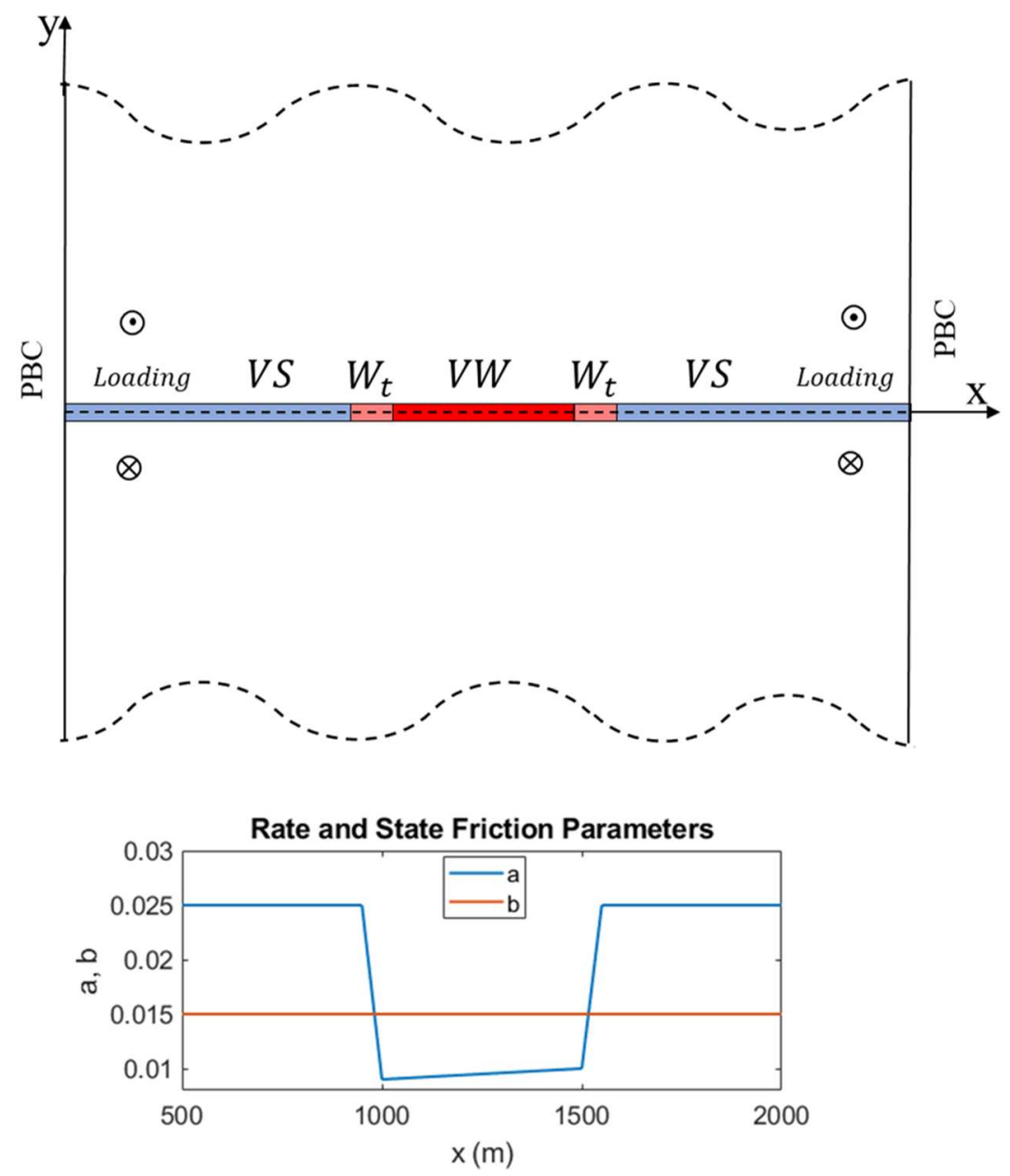

Fig-1: Model set-up and distribution of frictional parameters. Model geometry consists of rate-and-state fault embedded in elastoplastic bulk. Periodic Boundary Conditions (PBC) are applied at the lateral boundaries. $a$ and $b$ are non-negative dimensionless rate-and-state frictional parameters related to direct effect and state evolution respectively. Velocity weakening (VW) patch is associated with $a<b$ and velocity strengthening (VS) refers to $a>$ $b$. Transition from VS to VW happens over a length indicated as $W_{t}$. Out of plane loading in form of uniform plate

To solve the governing field equations, we employ a hybrid finite element-spectral boundary integral scheme which fully accounts for inertial effects during episodes of rapid slip by alternating between a quasi-dynamics algorithm during the interseismic period, and a dynamics solver during seismic slip. Spectral boundary integral enables truncating the computational domain by replacing the exterior homogeneous linear elastic half spaces with integral relation between the shear stress and displacement history. The finite element discretization includes the fault and the near-field nonlinear bulk. More details are outlined in Methods section and Supplementary Materials (Fig-S1). 

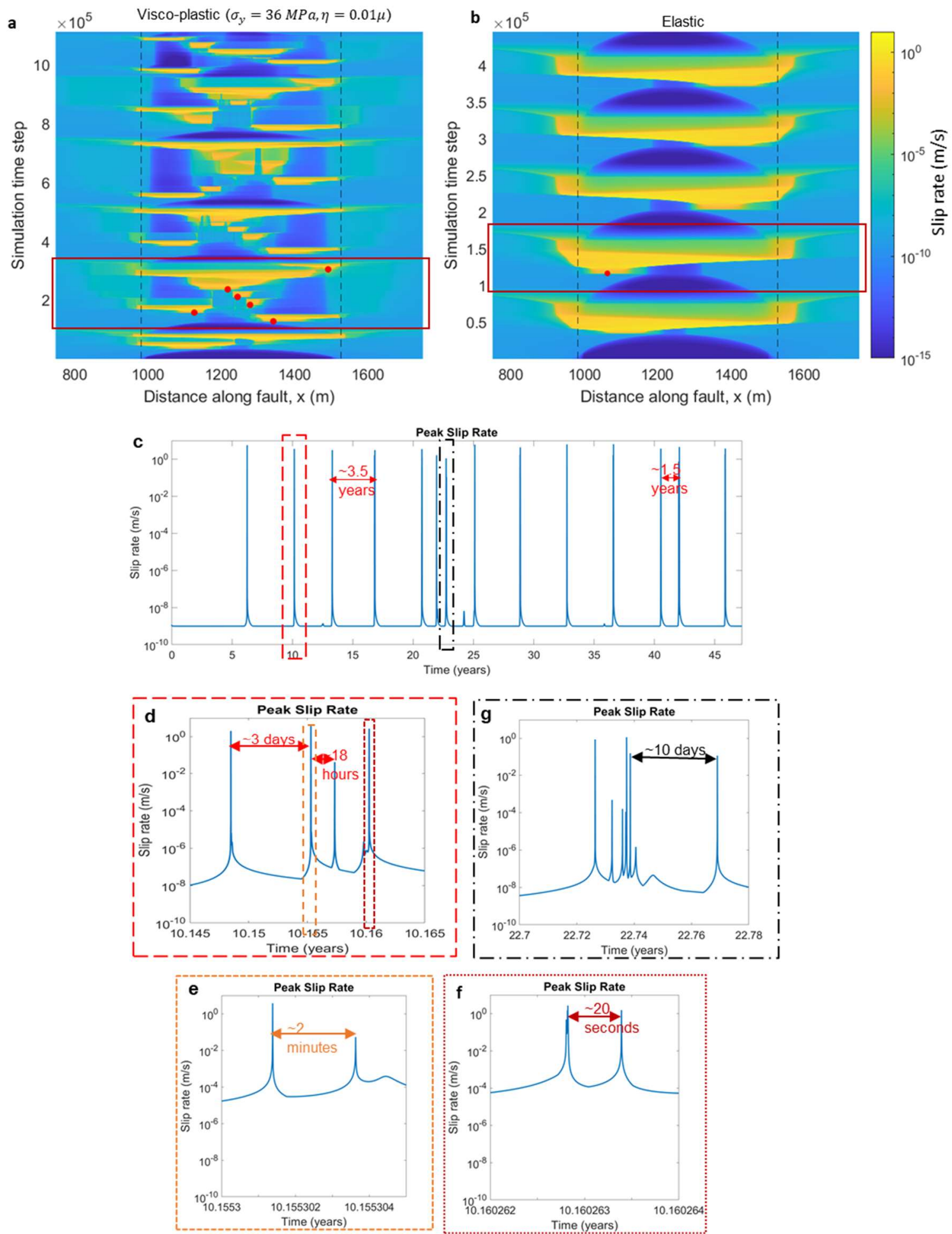

Fig-2: Slip rate evolution illustrating spatiotemporal clustering and hierarchical seismicity. a space-time evolution of slip rate for viscoplastic case plotted with simulation time step (not actual time) which shows multiple events with partial rupture in contrast to the elastic reference (shown in $\mathbf{b}$ ) with simple periodic cycles. Both $\mathbf{a}$ and $\mathbf{b}$ show results up to 25 years, and vertical dashed lines are drawn to indicate transition between VS and VW. Red circles indicate nucleation sites of different events in cluster-2 (within the rectangular box). $\mathbf{c}$ history of peak slip rate showing aperiodic seismic cycles with time interval in the scale of years. $\mathbf{d}$, $\mathbf{e}$, $\mathbf{f}$ zoomed-in view for cluster-2 showing temporal clustering of seismic events with time interval spanning days to seconds. $\mathrm{g}$ zoomed-in view of cluster-7 showing existence of temporal clustering. 


\section{Results}

\section{Hierarchical Spatiotemporal Clustering of Seismicity}

Fig-2 shows the evolution of slip rate with and without off-fault viscoplasticity. With off-fault visco-plasticity, seismic events are clustered in space and time with a mixture of partial ruptures and fault spanning events (Fig. 2a) whereas the elastic case generates simple periodic fault spanning earthquake events (Fig. 2b). During aseismic deformation, the active fault (VW region) remains almost locked demonstrated by the slip rate far below the loading $\left(V_{p}=10^{-9} \mathrm{~m} / \mathrm{s}\right)$ whereas the velocity strengthening (VS) region keeps creeping following the loading. During rapid rupture, the VW region moves with increasing slip rate as the rupture propagates. The arrest of rupture is indicated by subsequent decrease in slip rate. For the elastic simulation (Fig-2b), all seismic events rupture the entire VW region and get arrested after reaching the VS region. On the other hand, for the visco-plastic simulation (Fig-2a), not all events rupture the full length of the active fault. Rupture arrest is observed within VW region followed by nucleation of subsequent events highlighting the spatial migration and clustering. The red circles in Fig-2a mark the nucleation sites of different events in cluster-2. It shows that the nucleation site in this case does not necessarily exist near a rheological transition as in the elastic simulation (Fig. 2b). Rather the nucleation site jumps from one location to another during different events. That is, subsequent events nucleate within the VW region and not necessarily due to the expansion of creep fronts from the VS region as in Fig. $2 b$. The rupture arrest is associated with plastic strain accumulation and the arrest of partial rupture leaves stress concentration which in turn affects the nucleation and rupture characteristics of subsequent events.

Furthermore, while the elastic case is characterized by simple periodic events, the elastic-viscoplastic case exhibits temporal clustering of events and hierarchical seismicity. The time history of peak slip rate (Fig-2c) shows the aperiodic sequence of earthquake and aseismic slip that emerge with viscoplasticity. Before and after the seismic event, the peak slip rate is close to the applied plate rate $\left(V_{p}=10^{-9} \mathrm{~m} / \mathrm{s}\right)$ indicating slow aseismic slip. The occasional sharp increase of peak slip rate denotes the fast rupture of the active fault during the seismic event which apparently looks like vertical lines (Fig-2c). If we zoom in the time scale as shown in Fig- $2 \mathrm{~d}-2 \mathrm{~g}$ it reveals a richer structure spanning multiple time scales. Specifically, the apparent vertical lines in Fig. $2 \mathrm{c}$ are temporal clusters of seismicity encompassing multiple subevents with interevent time spanning days to seconds whereas the interevent time of each cluster of events as shown in Fig$2 \mathrm{c}$ is in the scale of years (1.5-3.5 years). Within cluster-2, for example, there are in total 6 events (Fig-2d, 2e,2f). The first two events occur within a few days apart (Fig-2d); the next event is found within next few minutes (Fig-2e). The last two events are found only in few seconds (Fig-2f). The temporal clustering prevails in subsequent clusters (Fig- $2 \mathrm{~g}$ ) even though the interevent times may vary from one cluster to another. This Russian-doll-like hierarchical seismicity is qualitatively similar to natural seismicity and suggest a crucial role of off-fault inelasticity in modulating rupture growth and arrest. 

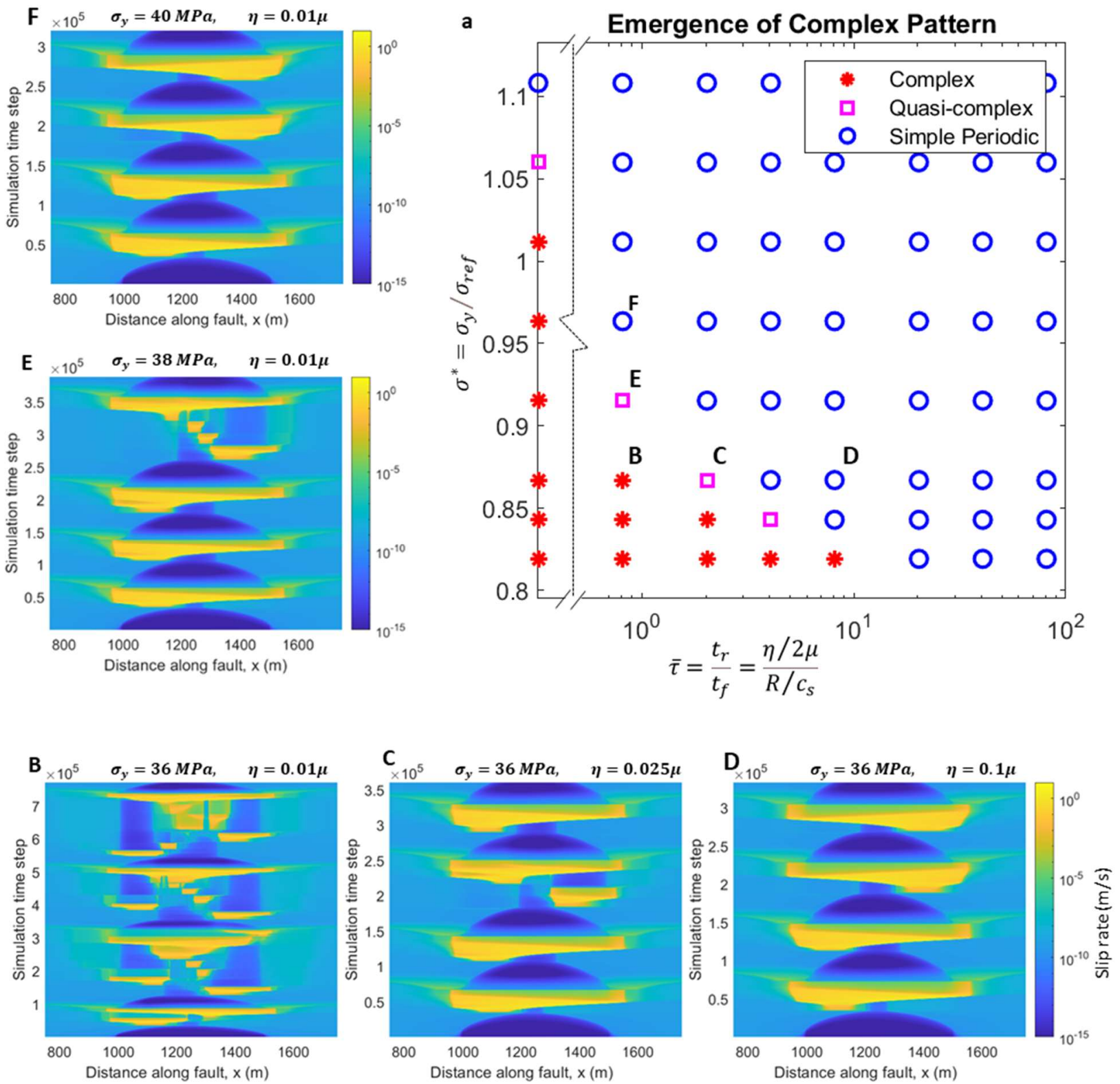

Fig-3: Emergence of complex seismic pattern depending on yield strength and viscosity. a summarizes seismicity patterns of several case studies by varying yield strength and viscosity. Yield strength $\left(\sigma_{y}\right)$ is normalized by a reference stress $\left(\sigma_{\text {ref }}=f_{0}+a \ln \left(V_{\text {seismic }} / V_{p}\right) \approx 41.5 \mathrm{MPa}\right)$ which is an estimation of peak stress for elastic case accounting direct effect in rate-and-state friction. Relaxation time is normalized by time scale associated with friction. Complex patterns are found with lower yield strength with lower range of viscosity. For a particular yield strength, decrease in viscosity leads to the emergence of spatiotemporal clustering as shown in B, C, and D. For a particular viscosity, decrease in yield strength leads to transition from periodic to quasicomplex and complex pattern (as shown in $\mathbf{E}$ and $\mathbf{F}$ ). Rate independent plasticity also shows transition from periodic to complex pattern when yield strength is decreased. 
To explore the robustness of the spatiotemporal complexity, we have carried out several simulations by varying bulk yield strength and viscosity. The viscosity is varied over two orders of magnitude from $\eta=0.01 \mu$ to $\eta=\mu$ which corresponds to varying the relaxation time, $t_{r}=$ $\eta / 2 \mu=0.005-0.5$ seconds. We assume a shear thinning rheology where we use adaptive relaxation during aseismic slow deformation (low strain rate) allowing viscosity to increase as the peak slip rate decreases while keeping the intended relaxation time fixed during coseismic deformation (high strain rate). The complexity in seismicity pattern is also found to be qualitatively similar when we use fixed relaxation time throughout aseismic and coseismic deformation. (Supplementary Materials, Fig-S2)

Different patterns of seismic cycles depending on yield strength and viscosity are summarized in Fig-3. We normalize bulk yield strength, $\sigma_{y}$, by a reference stress, $\sigma_{\text {ref }}=f_{0}+a \ln \left(V_{\text {seismic }} /\right.$ $\left.V_{p}\right) \approx 41.5 \mathrm{MPa}$, which estimates peak stress at a rupture tip within the elastic bulk accounting for the direct effect in rate-and-state friction. Relaxation time is also normalized by a time scale associated with friction. Since the change in frictional stress occurs over the length of process zone, $R=\frac{\mu L}{\sigma_{n} b}=21.36 \mathrm{~m}$, we choose the frictional time scale to be $t_{f}=R / c_{s}=0.0062 \mathrm{~s}$, where $c_{s}$ is the shear wave speed. For a given yield strength, when viscosity is decreased, we observe transition in the pattern of seismic cycles from simple periodicity to spatio-temporal complexity through a quasi-complex pattern. For instance, with yield strength, $\sigma_{y}=$ $36 \mathrm{MPa}\left(\sigma^{*} \approx 0.87\right)$, simple periodic patterns of seismic cycles are found for viscosity exceeding $0.05 \mu(\bar{\tau} \approx 4.05)$ but complex pattern is observed when viscosity is reduced to $0.01 \mu(\bar{\tau} \approx$ $0.81)$. For intermediate viscosities, quasi-complex event sequences may emerge. We distinguish complex pattern by spatiotemporal clustering of seismic events prevailing in almost every cluster whereas quasi-complex pattern is nearly periodic with occasional emergence of partial ruptures. The transition to complexity with the reduction in the viscosity may be explained as follows. The redistribution of stresses occurs over two time scales: the viscous relaxation time scale and the frictional time scale. At high viscosity, the relaxation time scale is long, and the stresses are redistributed rapidly over the frictional time scale similar to the elastic case. At low viscosity, the redistribution of stresses due to viscous relaxation is faster or as fast as with the frictional time scale and thus complexity emerge due to the competition of these two processes. However, the transition also depends on the yield strength. When yield strength is increased from $36 \mathrm{MPa}$ to $40 \mathrm{MPa}$ ( $\sigma^{*} \approx 0.87$ to 0.96 ) keeping the viscosity fixed such as $\eta=0.01 \mu(\bar{\tau} \approx 0.81)$, the seismic pattern becomes periodic through a quasi-complex pattern for the intermediate yield strength of $38 \mathrm{MPa}\left(\sigma^{*} \approx 0.92\right)$. This indicates that, for a particular viscosity, a decrease in yield strength, favoring higher accumulation of off-fault plasticity, gives rise to complex seismic pattern. In the limiting case of rate independent plasticity, complexity of earthquake sequences persists for all values of yield stress up to $\sigma^{*} \sim 1$ suggesting the critical role of off-fault plasticity in pinning the rupture tip and leading to hierarchical seismicity by limiting the ability of the crack to concentrate the stress beyond the yield limit as will be discussed shortly. 
a
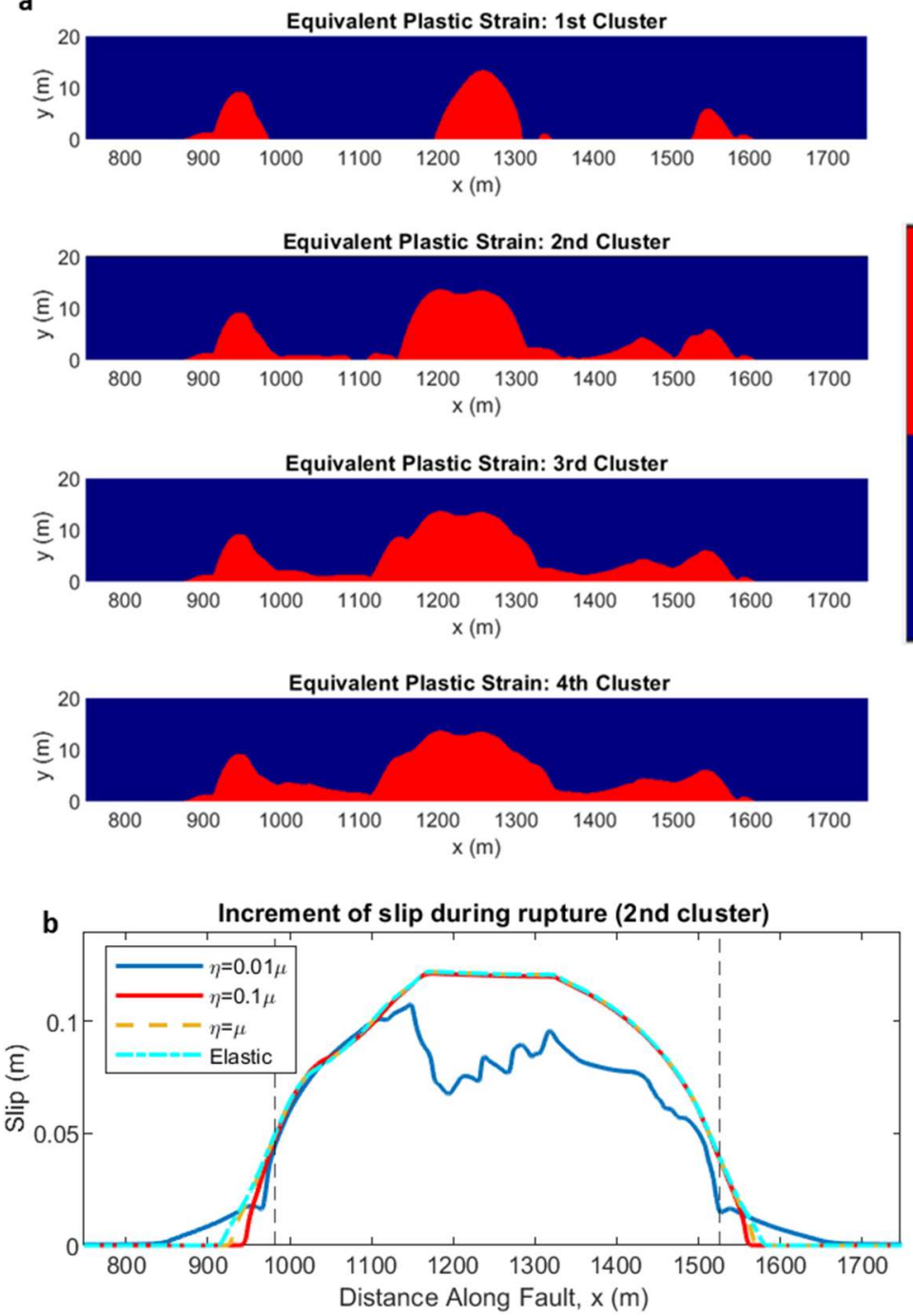

d

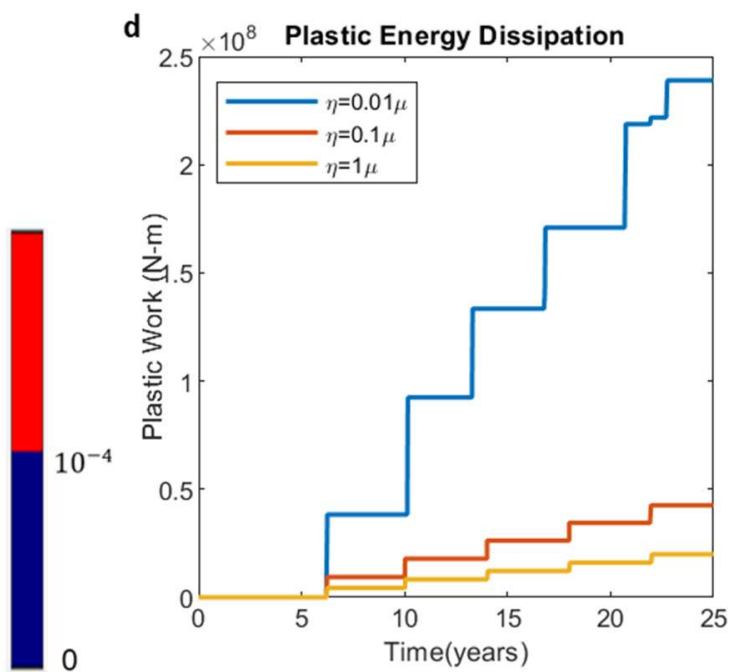

e

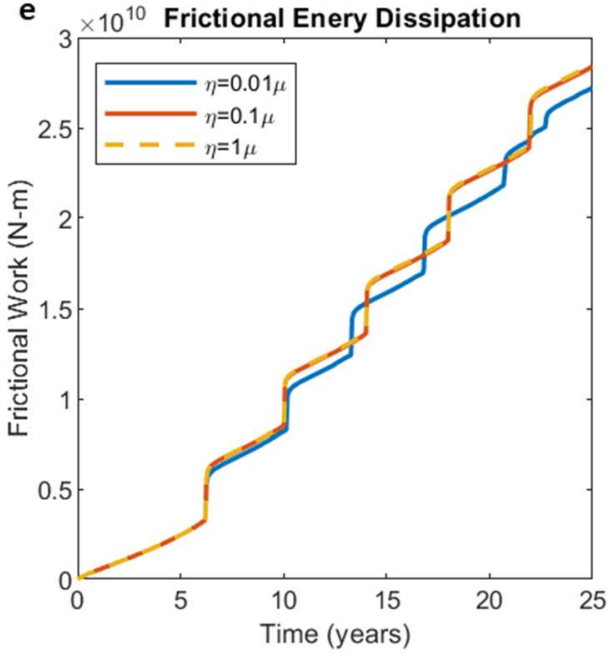

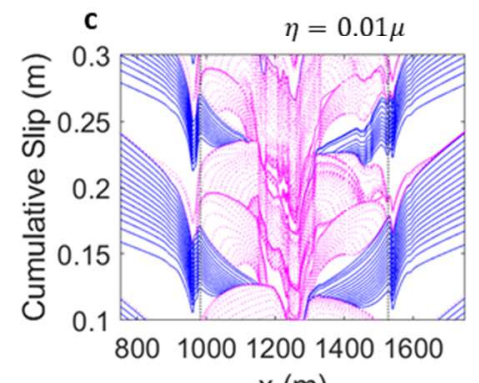

$x(\mathrm{~m})$

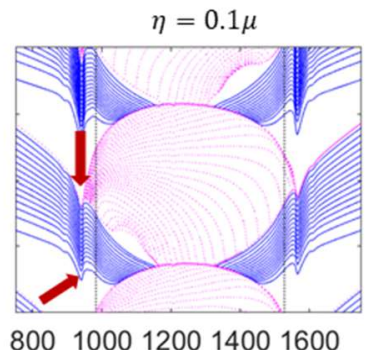

$\mathrm{x}(\mathrm{m})$

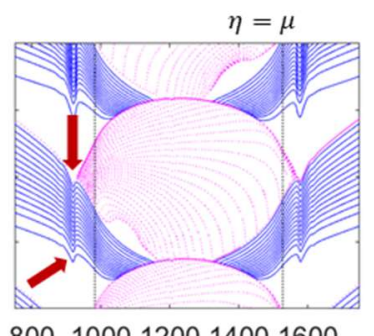

8001000120014001600

$x(\mathrm{~m})$

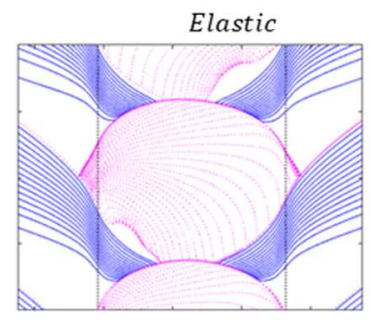

8001000120014001600

$x(m)$

Fig-4: Evolution of plastic strain and effect of viscosity. a Spatial distribution of equivalent plastic strain after cluster of events (yield strength $=36 \mathrm{MPa}$, viscosity, $\eta=0.01 \mu$ ). Plastic strain accumulation spreads along the fault with successive cluster. $\mathbf{b}$ shows increment of slip during rupture in the $2^{\text {nd }}$ cluster. $\mathbf{c}$ shows cumulative slip distribution where solid blue lines are plotted at every 3 months during aseismic slip and dotted magenta lines are at every 10 milliseconds during dynamic rupture. Lower viscosity case generates non-uniform slip while higher viscosity case is almost like elastic case except slip deficit near rupture arrest (as shown in $\mathbf{b}$ and $\mathbf{c}$ ). $\mathbf{d}$ Evolution of plastic energy dissipation indicates ceasing plasticity during aseismic slow deformation phase with considerable increase due to seismic events. e Frictional energy dissipation slowly increases during aseismic deformation due to slow creeping of VS region followed by rapid increase during coseismic slip. Relative change in frictional energy dissipation due to viscosity reduction is small but decrease in viscosity increases plastic energy dissipation significantly. 


\section{Evolution of off-fault inelasticity and fault slip}

A key contribution of this work is tracking the co-evolution of off-fault plastic strain with the accumulation of fault seismic and aseismic slip over long time scales. In Fig-4a spatial distribution of equivalent plastic strain shows plasticity primarily contained within relatively narrow region normal to the fault while rest of the bulk remains elastic. However, most notably, the accumulation of plastic strain is non-uniform along the fault. Plastic strain is higher in the region where rupture gets arrested. Multiple clusters of events lead to the non-uniform spreading of off-fault plastic strain parallel to the fault. Fig-4b shows the spatial distribution of slip accumulation within event cluster-2. The final slip distribution during this cluster shows that the VW region of the fault experienced extensive sliding compared to the VS region. Basically, slip deficit created by the slow creeping of the VS regions during aseismic deformation is compensated by the sliding of VW region through coseismic rupture. Higher viscosity cases $(\eta=$ $0.1 \mu$ and $\eta=\mu$ ) show smooth distribution of slip like the elastic case except small deficit near the rupture arrest region as indicated by the arrows in Fig-4c. On the other hand, there are oscillations in the spatial distribution of slip with lower viscosity $(\eta=0.01 \mu)$. Oscillations in the slip distribution arises from spatiotemporal clustering of seismic events with irregular nucleation, heterogenous propagation, and arrest of the rupture. For the low viscosity case, slip deficit is pervasive reflecting the significant partitioning of total deformation into fault slip and off-fault inelastic deformation of the bulk.

Plastic energy dissipation increases as viscosity decreases. When viscosity is reduced from $\eta=\mu$ to $\eta=0.1 \mu$, the plastic dissipation increases. However, the increase in plastic dissipation is much higher when viscosity is reduced from $\eta=0.1 \mu$ to $\eta=0.01 \mu$. Nonetheless, overall plastic energy dissipation is found to be very small compared to the frictional energy dissipation (attention to the scales of Fig- $4 \mathrm{~d}, 4 \mathrm{e}$ ). For instance, in cluster- 2 for $\eta=0.01 \mu$, plastic dissipation is below $3 \%$ of the corresponding frictional dissipation whereas plastic dissipation is below $0.6 \%$ of the corresponding frictional dissipation for the case of $\eta=0.1 \mu$. In both higher and lower range of viscosity, plastic dissipation is smaller than frictional dissipation. While, the off-fault inelastic dissipation remains small, plasticity plays a crucial role in altering the pattern of earthquake sequences through its impact on stress redistribution and its heterogeneity, depending on the parameters. This effect is intensified as the viscosity and yield strength decrease.

Specifically, the emergence of complex hierarchical seismicity is attributed to the plastic strain accumulation and resulting stress heterogeneity but not necessarily the fraction of energy dissipated globally in plastic work. Off-fault plastic strain pins the crack motion through acting as an additional local energy sink, adding to the breakdown energy at the crack tip, and increasing the effective toughness of the medium. Furthermore, off-fault plasticity reduces the stress concentration ahead of the crack tip by limiting the stress to a smaller value than what could be achieved for an elastic bulk. This stress limiting effect increases as the viscosity decreases, and for the rate-independent limit the stress ahead of the crack tip may not exceed the yield stress. This reduction in the ability to concentrate the stress leads to the arrest of the seismic rupture even within the velocity weakening patch and to the frequent emergence of partial ruptures. In the purely elastic case, only fault spanning ruptures emerge and the rupture arrest takes place in the velocity strengthening patch. In the elastic case, as the rupture propagates, the stress increases ahead of the rupture front, and the stress concentration effect, as measured for 
example by a stress intensity factor, increases with rupture propagation. For the elasticviscoplastic case, the stress may exceed the yield strength, depending on the loading rate and viscosity. The lower the viscosity is, the smaller this stress overshoot will be, and the more inelastic strain will accumulate. Similarly, the lower the yield strength, the higher visco-plastic strain. This inelastic response of the bulk introduces stress heterogeneity which affects rupture nucleation, propagation, and arrest. Fig-5 illustrates the state of stress close to the fault plane during coseismic rupture for the first event with elastic-perfectly plasticity bulk ( $\sigma_{y}=$ $\left.36 \mathrm{MPa}, \sigma^{*} \approx 0.87\right)$. Purely elastic bulk allows stress concentration ahead of the rupture tip which enables the rupture to propagate further. For elastoplastic bulk, however, off-fault plastic yielding limits the stress concentration which pins the crack tip and leads to rupture arrest. Furthermore, the jumping of the nucleation site that we reported in Fig. $2 \mathrm{a}$ is a direct consequence of the generation of off-fault plastic strain ahead of the crack tip. As the rupture arrests and this plastic zone forms, the stress must increase on the fault beyond the plastic zone in order to nucleate a new event within an already stress relaxed region. As a result, the sequence of events appears segmented and the nucleation sites move from one location to another guided by the plastic strain distribution.
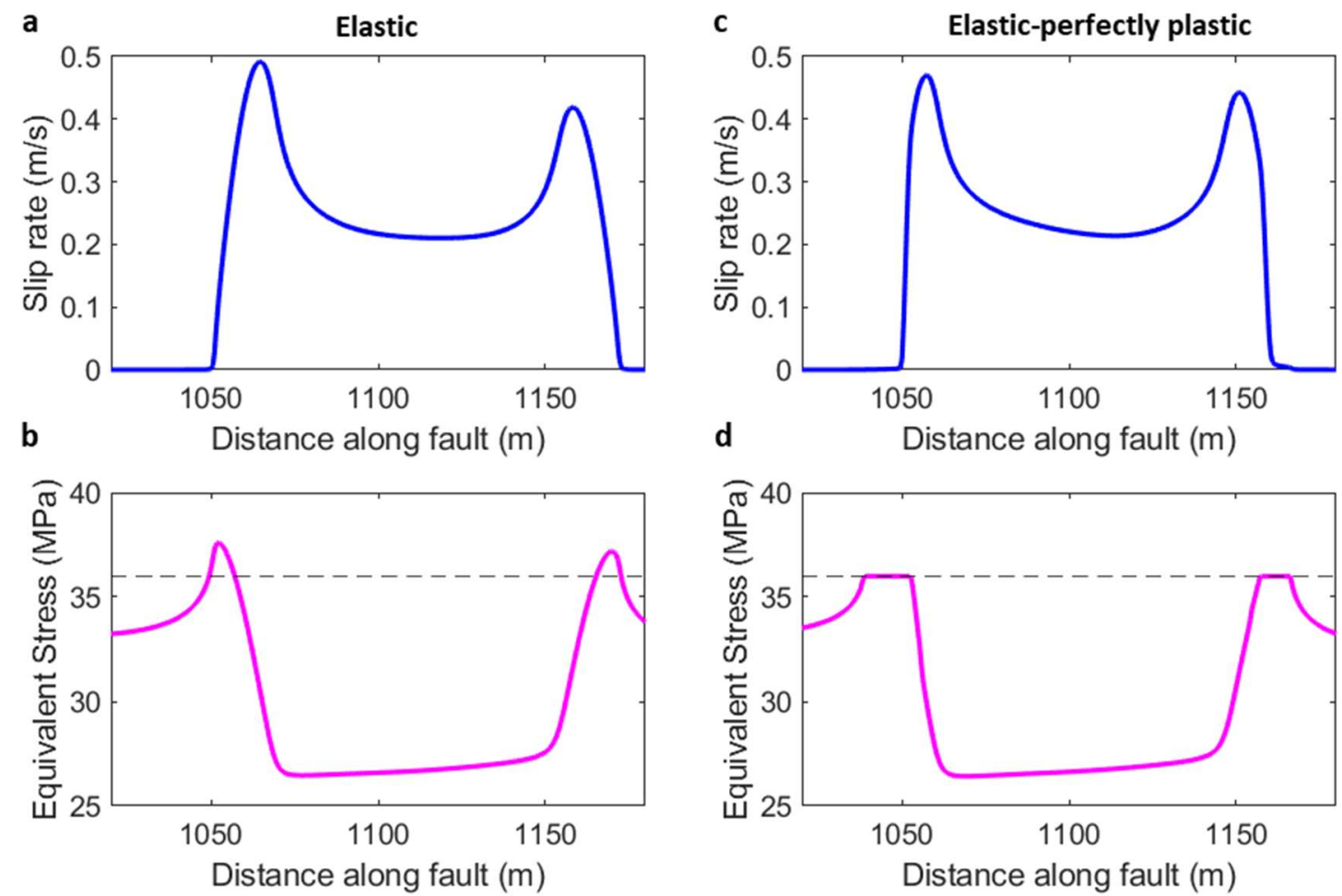

Fig-5: Comparison of the state of stress for elastic and elastic-perfectly plastic bulk. $\mathbf{a}$ and $\mathbf{b}$ respectively show spatial distribution of slip rate and equivalent stress $\left(\sqrt{\sigma_{x z}^{2}+\sigma_{y z}^{2}}\right)$ close to the fault plane for elastic case where stress concentration occurs ahead of the rupture tip. Horizontal dashed line in $\mathbf{b}$ and $\mathbf{d}$ is drawn to mark the bulk yield strength. For elastic-perfectly plastic bulk, stress ahead of the rupture tip is bounded by the yield strength due to plastic yielding of the bulk. This reduced stress concentration with off-fault plasticity leads to rupture arrest. 


\section{Discussion}

In this work, we showed how the co-evolution of fault slip and off-fault plasticity may lead to the emergence of spatio-temporal complexity of sequences of earthquakes and aseismic slip. This is a new mechanism for dynamic heterogeneity that has not been highlighted before and suggest the need for monitoring both on-fault and off-fault processes over multiple spatial and temporal scales as they are tightly coupled with strong feedback loops.

The novelty of our hybrid numerical scheme enabled us to perform high resolution modeling of the complex nonlinear problem covering all phases of the seismic cycle from aseismic creep to fully inertial dynamic rupture while accounting for bulk inelasticity. This enabled us to map the fault response over a wide range of parameters that were not accessible before. Our work suggests that two non-dimensional parameters control the transition from simple periodicity to spatio-temporal complexity; namely the ratio of the yield stress to the elastic peak stress and the ratio of the viscous time scale to the frictional time scale. As viscosity decreases or the yield strength decreases, the off-fault plastic strain increases, and this coevolution of off-fault bulk inelasticity and on-fault slip leads to hierarchical seismicity in time and partial ruptures in space. This sheds light on a new mechanism for modulating the sequence of earthquake and aseismic slip. The pinning effect of off-fault plasticity leads to rupture arrest and jumping of the nucleation site. This intermittent stick-slip nature is also observed in other applications at different scales. For example, plane strain elastic-perfectly plastic medium is found to host intermittent crack propagation where crack is pinned by plastic zone and finite jump in nucleation is observed (Brach et al., 2019). Intermittent stick-slip behavior is also observed in crystal plasticity through avalanches in stress-strain response of a micropillar assisted by dislocation pinning (Sethna et al., 2017). Thus, plasticity is emerging as a unifying mechanism for modulating instabilities from micro to macro scales and with or without inertia effects.

Even though plastic dissipation is a small fraction of the total energy budget, its impact on the rupture dynamics is amplified by its feedback on stress heterogeneity. Global quantities like energy balance may not reveal the full picture of the source physics and there is a need for investigation of small-scale physics in complex fault zones due to their evolutionary nature and feedback loops they introduce. Besides, investigation with wider parameter space including variation in bulk yield strength and viscosity reveals the dependency of the pattern of seismic cycles on off-fault inelastic material properties. This indicates the need for accurately characterizing the nonlinear rheology of rocks.

Since our model results are parametrized by a set of non-dimensional parameters, the results are relevant to earthquakes at different scales. Specifically, off-fault plasticity may act as a selflimiting process for the growth of induced seismicity and hence may contribute to modulating seismicity at injection sites. The complexity reported here does not require long faults (Cattania, 2019; Barbot, 2019) or rheological heterogeneity (Kaneko et al., 2010). Rather, it emerges naturally as a dynamic process due to the feedback between off-fault plasticity and on-fault stress heterogeneity which may occur at any scale. This suggests that accounting for inelastic processes may be necessary even for small earthquakes and short faults. 
A limitation of this study includes the lack of consideration of coseismic variation in elastic moduli as well as interseismic healing which has been widely documented and are suggested by some studies (Ben-Zion \& Lyakhovsky, 2019; Ben-Zion \& Huang, 2002; Tenthorey \& Cox, 2006; Griffith et al., 2012). Furthermore, extending the model to 2D plane strain and full 3D may enrich the response through incorporation of normal stress changes and asymmetric generation of damage and plasticity. We plan to incorporate these additional feedback mechanisms in future work.

\section{Methods}

We use an alternating quasi-dynamics and fully inertial dynamics approach to capture full time history of the earthquake sequence including both slow aseismic creep and fast dynamic rupture. Quasi-dynamics approach approximate inertia through radiation damping whereas dynamics approach fully accounts inertia. The switching between the two solvers is enabled based on a threshold slip rate of $0.01 \mathrm{~m} / \mathrm{s}$ below which radiation damping contribution $\left(\mu \mathrm{V} / 2 c_{s}\right)$ is very small ( $\sim 0.1 \%$ of the quasi-static shear stress with elastic bulk). During aseismic slow deformation, we solve a series of static equilibria to get fault traction for a given slip constraint. Then radiation damping is used while satisfying fault boundary condition. Quasi-dynamics approach involves adaptive time increment based on slip rate (Lapusta et al.,2000; Abdelmeguid et al., 2019). On the other hand, explicit dynamics approach fully accounts for inertia effects and integrates the equations of motion with fixed time increment governed by CFL condition. In the dynamics formulation, we use traction-at-split-node (TSN) to compute fault traction as function of slip rate (Moczo et al, 2007; Setare \& Elbanna, 2017).

In spatial discretization, we employ a hybrid scheme (Albertini et al., 2021, Abdelmeguid et al., 2019) combining finite element and spectral boundary integral. A narrow strip containing the fault and potential elastoplastic bulk is discretized with finite element (FEM) and the rest of the homogeneous linear elastic half-spaces are replaced by spectral boundary integral equations (SBI) A sketch of the hybrid scheme set-up is shown in Supplementary Materials (Fig-S1). This hybrid scheme truncated the computational domain significantly and provide an exact transparent boundary condition for wave propagation. We use a mesh size $(d x=d y=0.5 \mathrm{~m})$ such that the characteristic length scale defined by the static estimate of the process zone size, $R=\frac{\mu L}{\sigma_{n} b}=$ $21.36 \mathrm{~m}$ is resolved by around 40 elements. We use radial return algorithm with viscous regularization (Simo \& Hughes, 2006) to update the stresses. During coseismic phase, time increment is smaller than relaxation time by at least one order of magnitude which makes the relaxation time scale well resolved by simulation time increment. During aseismic slow deformation, we adopt relaxation time following the simulation time increment so that relaxation time is sufficiently resolved by the simulation time increment. Results do not qualitatively change when we use fixed relaxation time throughout (Supplementary Materials, Fig-S2). Besides, we carry out fully quasi-dynamic simulation where we find the complex seismicity pattern (Supplementary Materials, Fig-S3). 


\section{Acknowledgement}

The authors acknowledge support from the Southern California Earthquake Center through a collaborative agreement between NSF. Grant Number: EAR0529922 and USGS. Grant Number: 07HQAG0008 and the National Science Foundation CAREER award No. 1753249 for modeling complex fault zone structures. This material is also based upon work partially supported by the Department of Energy under Award Number DE-FE0031685 to investigate spatio-temporal complexity of induced earthquakes. 


\section{References}

Abdelmeguid, M., Ma, X., \& Elbanna, A. (2019). A novel hybrid finite element-spectral boundary integral scheme for modeling earthquake cycles: Application to rate and state faults with low-velocity zones. Journal of Geophysical Research: Solid Earth, 124(12), 12854-12881.

Albertini, G., Elbanna, A., \& Kammer, D. S. (2021). A three-dimensional hybrid finite element--spectral boundary integral method for modeling earthquakes in complex unbounded domains. International Journal for Numerical Methods in Engineering, 122, 6905-6923.

Allison, K. L., \& Dunham, E. M. (2021). Influence of Shear Heating and Thermomechanical Coupling on Earthquake Sequences and the Brittle-Ductile Transition. Journal of Geophysical Research: Solid Earth, 126(6), e2020JB021394.

Allison, K. L., \& Dunham, E. M. (2018). Earthquake cycle simulations with rate-and-state friction and power-law viscoelasticity. Tectonophysics, 733, 232-256.

Ampuero, J. P., \& Rubin, A. M. (2008). Earthquake nucleation on rate and state faults-Aging and slip laws. Journal of Geophysical Research: Solid Earth, 113(B1).

Barbot, S. (2019). Slow-slip, slow earthquakes, period-two cycles, full and partial ruptures, and deterministic chaos in a single asperity fault. Tectonophysics, 768, 228171.

Ben-Zion, Y., \& Lyakhovsky, V. (2019). Representation of seismic sources sustaining changes of elastic moduli. Geophysical Journal International, 217(1), 135-139.

Ben-Zion, Y., \& Huang, Y. (2002). Dynamic rupture on an interface between a compliant fault zone layer and a stiffer surrounding solid. Journal of Geophysical Research: Solid Earth, 107(B2), ESE-6.

Ben-Zion, Y., \& Rice, J. R. (1997). Dynamic simulations of slip on a smooth fault in an elastic solid. Journal of Geophysical Research: Solid Earth, 102(B8), 17771-17784.

Brach, S., Tanné, E., Bourdin, B., \& Bhattacharya, K. (2019). Phase-field study of crack nucleation and propagation in elastic-perfectly plastic bodies. Computer Methods in Applied Mechanics and Engineering, 353, 44-65.

Cattania, C., \& Segall, P. (2021). Precursory slow slip and foreshocks on rough faults. Journal of Geophysical Research: Solid Earth, 126(4), e2020JB020430.

Cattania, C. (2019). Complex earthquake sequences on simple faults. Geophysical Research Letters, 46(1718), 10384-10393.

Dieterich, J. H. (1979). Modeling of rock friction: 1. Experimental results and constitutive equations. Journal of Geophysical Research: Solid Earth, 84(B5), 2161-2168.

Duru, K., Allison, K. L., Rivet, M., \& Dunham, E. M. (2019). Dynamic rupture and earthquake sequence simulations using the wave equation in second-order form. Geophysical Journal International, 219(2), 796-815.

Dunham, E. M., Belanger, D., Cong, L., \& Kozdon, J. E. (2011). Earthquake ruptures with strongly rateweakening friction and off-fault plasticity, Part 1: Planar faults. Bulletin of the Seismological Society of America, 101(5), 2296-2307. 
Dunham, E. M., Belanger, D., Cong, L., \& Kozdon, J. E. (2011). Earthquake ruptures with strongly rateweakening friction and off-fault plasticity, Part 2: Nonplanar faults. Bulletin of the Seismological Society of America, 101(5), 2308-2322.

Erickson, B. A., Dunham, E. M., \& Khosravifar, A. (2017). A finite difference method for off-fault plasticity throughout the earthquake cycle. Journal of the Mechanics and Physics of Solids, 109, 50-77.

Gabriel, A. A., Ampuero, J. P., Dalguer, L. A., \& Mai, P. M. (2013). Source properties of dynamic rupture pulses with off-fault plasticity. Journal of Geophysical Research: Solid Earth, 118(8), 4117-4126.

Griffith, W. A., Mitchell, T. M., Renner, J., \& Di Toro, G. (2012). Coseismic damage and softening of fault rocks at seismogenic depths. Earth and Planetary Science Letters, 353, 219-230.

Hajarolasvadi, S., \& Elbanna, A. E. (2017). A new hybrid numerical scheme for modelling elastodynamics in unbounded media with near-source heterogeneities. Geophysical Journal International, 211(2), 851-864.

Kaneko, Y., Avouac, J. P., \& Lapusta, N. (2010). Towards inferring earthquake patterns from geodetic observations of interseismic coupling. Nature Geoscience, 3(5), 363-369.

Lapusta, N., Rice, J. R., Ben-Zion, Y., \& Zheng, G. (2000). Elastodynamic analysis for slow tectonic loading with spontaneous rupture episodes on faults with rate-and state-dependent friction. Journal of Geophysical Research: Solid Earth, 105(B10), 23765-23789.

Lapusta, N., \& Rice, J. R. (2003). Nucleation and early seismic propagation of small and large events in a crustal earthquake model. Journal of Geophysical Research: Solid Earth, 108(B4).

Moczo, P., Robertsson, J. O., \& Eisner, L. (2007). The finite-difference time-domain method for modeling of seismic wave propagation. Advances in geophysics, 48, 421-516.

Ruina, A. (1983). Slip instability and state variable friction laws. Journal of Geophysical Research: Solid Earth, 88(B12), 10359-10370.

Sethna, J. P., Bierbaum, M. K., Dahmen, K. A., Goodrich, C. P., Greer, J. R., Hayden, L. X., ... \& Zapperi, S. (2017). Deformation of crystals: Connections with statistical physics. Annual Review of Materials Research, 47, 217-246.

Simo, J. C., \& Hughes, T. J. (2006). Computational inelasticity (Vol. 7). Springer Science \& Business Media.

Tal, Y., Hager, B. H., \& Ampuero, J. P. (2018). The effects of fault roughness on the earthquake nucleation process. Journal of Geophysical Research: Solid Earth, 123(1), 437-456.

Templeton, E. L., \& Rice, J. R. (2008). Off-fault plasticity and earthquake rupture dynamics: 1. Dry materials or neglect of fluid pressure changes. Journal of Geophysical Research: Solid Earth, 113(B9).

Tenthorey, E., \& Cox, S. F. (2006). Cohesive strengthening of fault zones during the interseismic period: An experimental study. Journal of Geophysical Research: Solid Earth, 111(B9).

Thakur, P., Huang, Y., \& Kaneko, Y. (2020). Effects of low-velocity fault damage zones on long-term earthquake behaviors on mature strike-slip faults. Journal of Geophysical Research: Solid Earth, 125(8), e2020JB019587.

Viesca, R. C., Templeton, E. L., \& Rice, J. R. (2008). Off-fault plasticity and earthquake rupture dynamics: 2. Effects of fluid saturation. Journal of Geophysical Research: Solid Earth, 113(B9). 


\section{Supplementary Materials}

Table S1: List of parameters used in the simulations

\begin{tabular}{|lcc|}
\hline \multicolumn{1}{c}{ Parameter } & Symbol & Values \\
\hline Effective normal stress on fault & $\sigma_{n}$ & $50 \mathrm{MPa}$ \\
\hline Critical slip distance & $L$ & $500 \times 10^{-6} \mathrm{~m}$ \\
\hline Plate rate & $V_{p}$ & $10^{-9} \mathrm{~m} / \mathrm{s}$ \\
\hline Reference Slip rate & $V_{0}$ & $10^{-6} \mathrm{~m} / \mathrm{s}$ \\
\hline Initial slip rate & $V_{\text {init }}$ & $10^{-9} \mathrm{~m} / \mathrm{s}$ \\
\hline Reference friction coefficient & $f_{0}$ & 0.6 \\
\hline Shear wave speed & $c_{s}$ & $3464 \mathrm{~m} / \mathrm{s}$ \\
\hline Shear modulus & $\mu$ & $32.038 \mathrm{GPa}$ \\
\hline Yield strength & $\sigma_{y}$ & Variable \\
\hline Viscosity & $\eta$ & Variable \\
\hline
\end{tabular}

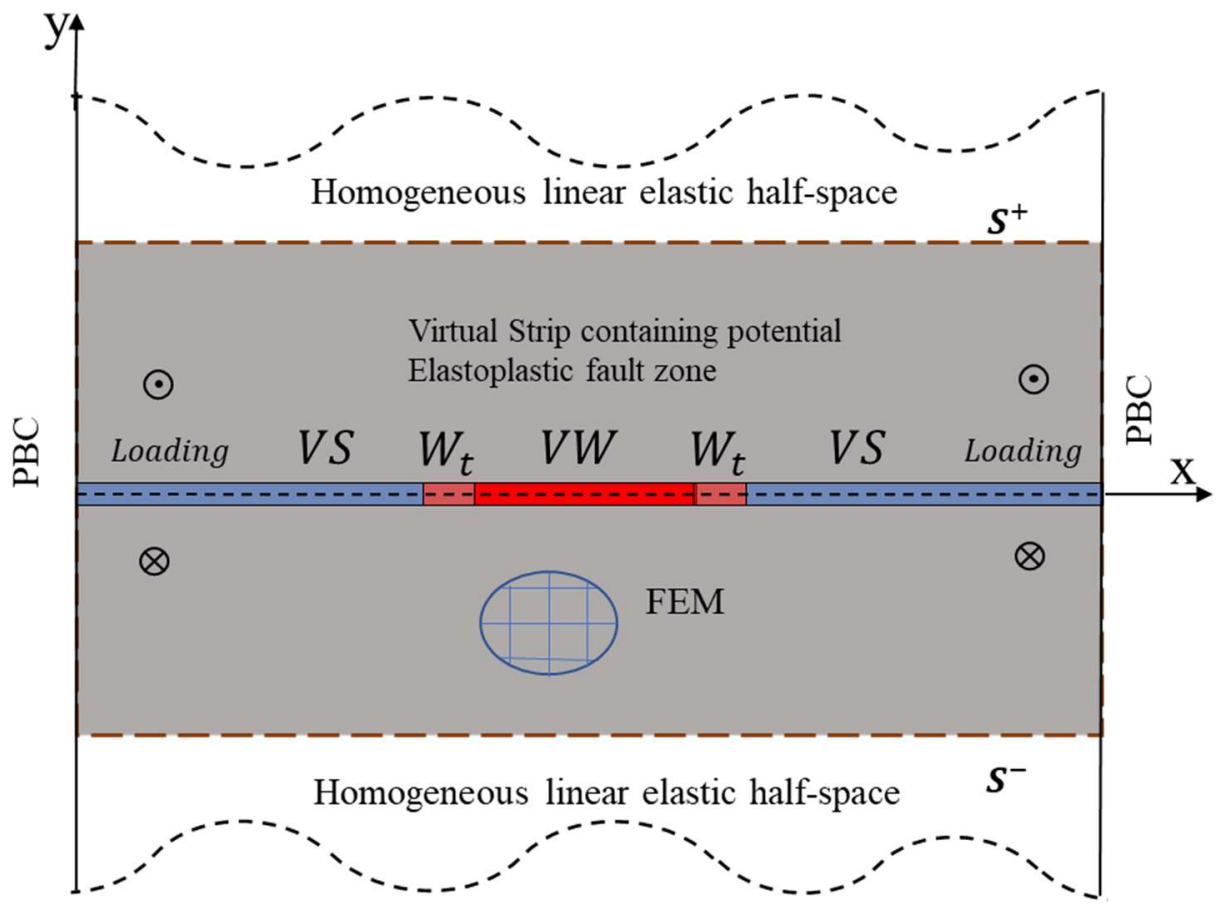

Fig-S1: Hybrid scheme set-up. Narrow virtual strip containing fault and potential elastoplastic bulk is discretized with FEM. Spectral Boundary Integral (SBI) replaces homogeneous linear elastic half spaces at the virtual boundaries $\left(S^{+}\right.$and $\left.S^{-}\right)$. Periodic Boundary Conditions (PBC) are applied at the lateral boundaries. 


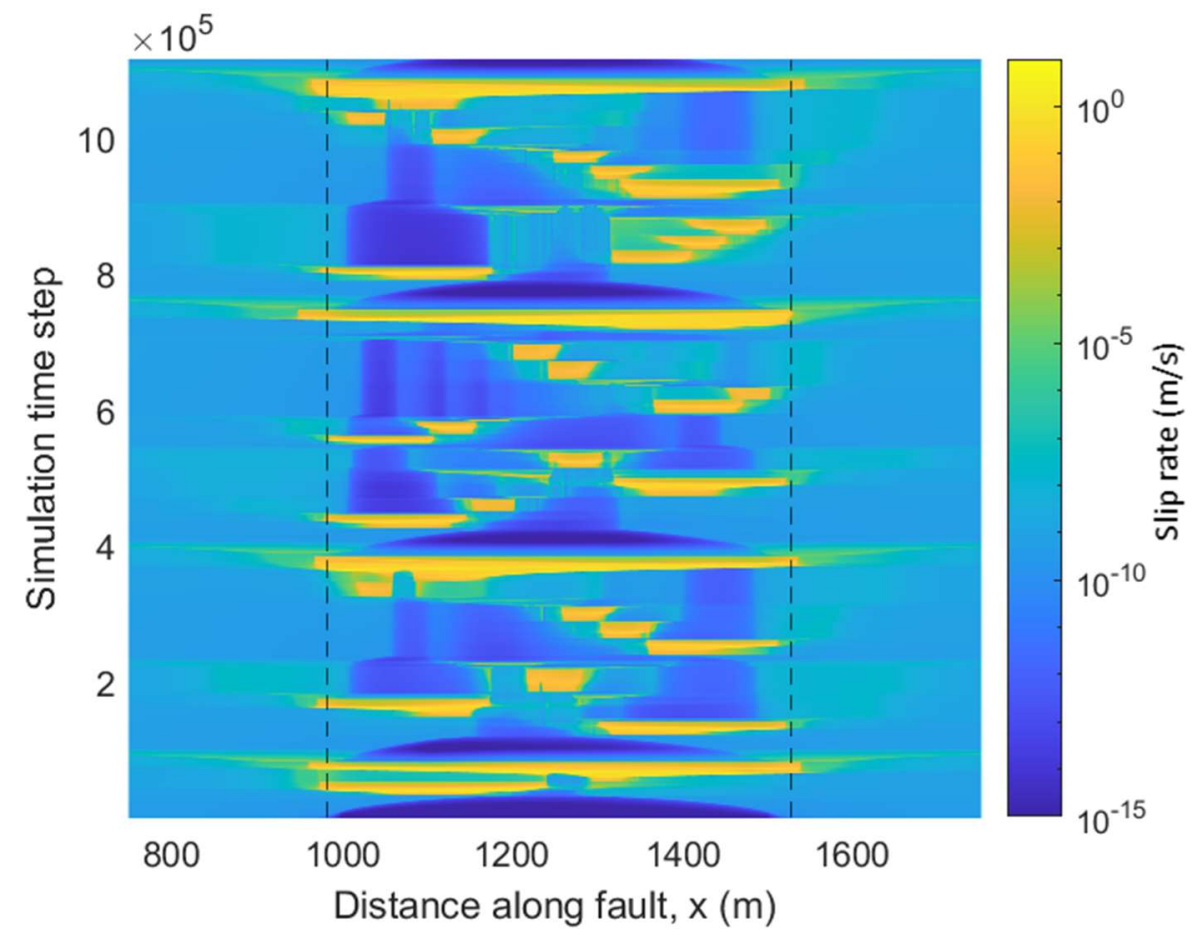

Fig-S2: Spatio-temporal distribution of slip rate for fully-dynamics simulation with fixed relaxation (yield strength $=36 \mathrm{MPa}$, viscosity, $\eta=0.01 \mu$ ). Temporal clustering and partial rupture are observed which are qualitatively similar to the case with adaptive relaxation (Fig-2a in main text). Adaptive relaxation ensures that relaxation time scale is well resolved both in dynamics and quasi-dynamics phases assuming a shear thinning rheology where viscosity is varied during quasi-dynamics depending on peak slip rate and keeping the intended viscosity constant during dynamics. The features of complex seismicity pattern are qualitatively similar in both cases. 


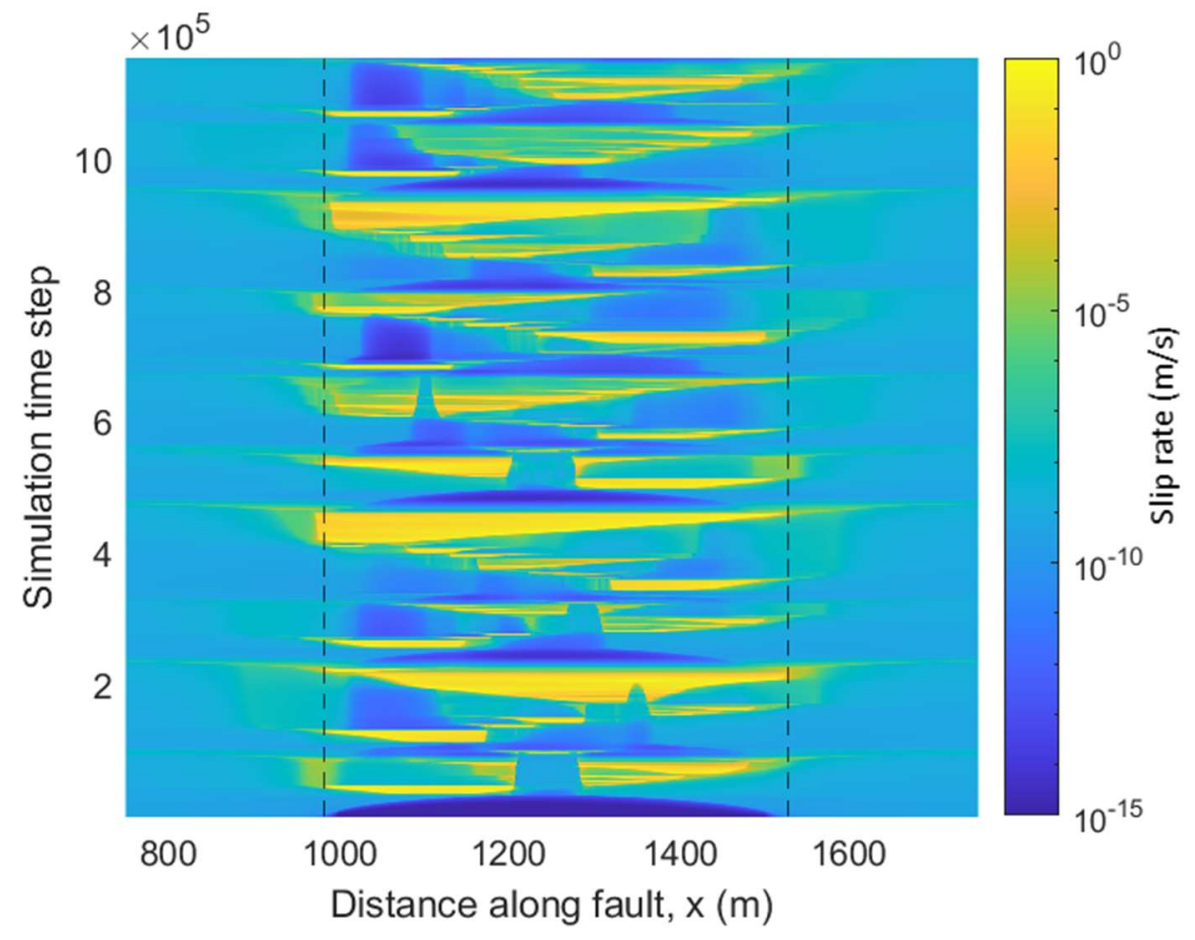

Fig-S3: Spatio-temporal distribution of slip rate for quasi-dynamics simulation with adaptive relaxation (yield strength $=36 \mathrm{MPa}$, viscosity, $\eta=0.01 \mu$ ) where inertia is approximated with radiation damping. Features of complex seismicity pattern including temporal clustering and partial rupture are observed which are qualitatively similar to fully-dynamics simulation. Unlike fully-dynamics simulation, there is no switching between two alternating solvers in case of fully quasi-dynamics simulation. Therefore, these qualitatively similar results suggest that the complex seismicity pattern is not a possible artifact of switching between solvers. 